\title{
Visual dissociation of digitized photographs
}

\author{
PAMELA J. BISHOP and HELENE INTRAUB \\ University of Delaware, Newark, Delaware
}

\begin{abstract}
Visual dissociation occurs when a visual marker from one display in a rapidly presented sequence (e.g., 9 item/sec) is perceived as having occurred in a temporally adjacent display. Three experiments that evaluated the application of computer graphics technology to study this phenomenon with digitized color photographs are reported. The primary concern was that visible phosphor persistence might artificially increase the frequency of integration errors. In Experiment 1, visible phosphor persistence was assessed using a multiple-repetition shutter test to determine which stimulus conditions did not yield reportable persistence. On the basis of these results, visual dissociation performance when the same color photographs were presented using mechanical 16-mm projection (as in previous research) and when they were presented on a computer monitor were compared in Experiments 2 and 3 . The results supported computer application, in that computer presentation yielded the same pattern of errors and accuracy levels as did research using mechanical projection.
\end{abstract}

The purpose of our research was to determine whether a computer graphics system could be used to conduct research on visual dissociation when digitized color photographs served as stimuli. Visual dissociation research requires rapid serial visual presentation (RSVP; e.g., 9 pictures/second). Subjects search each sequence for a picture that contains a specified feature. Until now, research of this type has been conducted using single-frame photography and 16-mm data analyzer projectors (e.g., Intraub, 1985, 1989; Potter, 1976). Our primary concern was whether visible phosphor persistence on the monitor would limit the usefulness of computer application in this research area. Various forms of visible persistence and iconic representation have been clearly established (e.g., Burr, 1980; Coltheart, 1980; DiLollo \& Dixon, 1988; Farrell, 1984; Farrell, Pavel, \& Sperling, 1990; Loftus, Duncan, \& Gehrig, 1992; Neisser, 1967). The danger is in confusing the effects of phosphor persistence on the screen with persistence in the visual/cognitive system (see Irwin, Yantis, \& Jonides, 1983; Jonides, Irwin, \& Yantis, 1983; Rayner \& Pollatsek, 1983).

Three approaches were taken. First, a shutter test was conducted to determine whether we could detect visible phosphor persistence with our stimuli. Second, in an attempt to eliminate or minimize persistence, we tested the effectiveness of following each picture with a white "bleaching" field for one noninterlaced video frame. The third approach involved direct comparisons of subjects' performance when the same pictorial sequences were presented mechanically, using a 16-mm data analyzer projector, and when they were presented on a color monitor.

This research was supported in part by National Science Foundation Grant BNS 9011015 to H.I. We thank our programmers, Karl Aldinger and Eric Moyer, for their valuable contributions to this research. Correspondence should be addressed to $\mathrm{H}$. Intraub, Department of Psychology, University of Delaware, Newark, DE 19716 (e-mail: intraub@ strauss.udel.edu).

\section{Visual Dissociation}

Visual dissociation (also referred to as "temporal migration") is a perceptual error that occurs when stimuli are presented in rapid succession (e.g., 9-20 items/sec) in the same spatial location (e.g., Gathercole \& Broadbent, 1984; Intraub, 1985, 1989; Lawrence, 1971; McLean, Broadbent, \& Broadbent, 1983). The task typically requires the subject to view a rapidly presented stream of stimuli (usually alphanumeric symbols or words) and to report the stimulus containing a specified target characteristic. The phenomenon occurs when subjects confidently report the target characteristic as occurring not on the stimulus, but on a temporally adjacent item. For example, using color photographs of objects and a presentation rate of 9 items/sec, Intraub $(1985,1989)$ showed that a black frame that surrounded one picture in a 12-picture sequence was frequently and confidently reported as being on the immediately preceding or immediately following picture in the sequence.

Intraub (1989) proposed a model of the early stages of picture processing that provides a possible explanation of this phenomenon. A central component of the model is a very short-term visual/conceptual store (Avons \& Phillips, 1980; Potter, 1976). Integration of features and comprehension of the display take place at this stage of processing. There is evidence to suggest that under conditions of rapid continuous presentation (faster than 3 items/ sec), more than one picture at a time may be held in this very short-term store (Intraub, 1984, 1989). It is under conditions of simultaneous processing that temporal migration errors are likely to occur.

According to this view, migration of a component occurs when display integration time is long relative to presentation rate. When this is the case, integration of the components of one display is not complete at the time that the next display enters the store. Longer integration times would be expected for displays that were more complex or had unfamiliar attributes. Thus, to obtain the same 
level of frame migration among different types of stimuli, a presentation rate used for stimuli that are relatively easy to identify must be faster than that used for stimuli that are relatively difficult-for example, numbers as compared with colored objects (Intraub, 1985) and letters as compared with words (Gathercole \& Broadbent, 1984).

Finally, the direction of migration (preceding or following picture) is apparently determined to some degree by the speed of target detection (Intraub, 1989). When the target is detected rapidly, it is more likely to become integrated with the previous picture ( -1 error). When it is detected a little more slowly, it is more likely to become integrated with the next new picture that enters the store $(+1$ error). Intraub (1989) reported that reaction times to target detection were significantly faster on trials in which subjects had made a high-confidence -1 error than they were on ones in which they had made a high-confidence +1 error. The difference in detection time was about $26 \mathrm{msec}$.

\section{Application of Computer Technology}

The importance of computer technology to research on picture perception is not limited to the obvious benefit of eliminating the tedious task of single-frame photography. It allows a more fine-grained manipulation of presentation rate than does mechanical projection, and computer graphics technology allows digitized photographs to be edited in ways that are difficult to accomplish with photography alone.

There are several potential problems facing researchers who use computer graphics technology to conduct research requiring precise tachistoscopic presentations, particularly with complex stimuli that can take a relatively long time to generate on the computer. Different approaches have been described for presenting visual information for precise tachistoscopic durations (e.g., Dixon, 1991; Paredes, Miller, \& Creeger, 1990). In our research, using an ATVista graphics board $(4 \mathrm{Mb})$ and $C$ programming, up to 15 digitized images can be stored in the video array, they can be presented in any order, and each can be presented for as little as a single noninterlaced video frame. A primary concern for us was the possibility of visible phosphor persistence, which raises an obvious problem - that is, determining whether the subject's error in reporting the conjunction of visual events reflects integration errors within the human visual system or integrated composites of stimuli within the computer display (see Irwin et al., 1983; Jonides et al., 1983; Rayner \& Pollatsek, 1983).

\section{EXPERIMENT 1}

The purpose of Experiment 1 was to determine whether (1) subjects could detect the presence of a stimulus picture on the basis solely of phosphor persistence, and (2) visible phosphor persistence could be eliminated by introducing a white "bleaching" field for one noninterlaced video frame following each picture.
To address the first question, the digitized photographs were alternated with a blank field (either gray or black). Subjects viewed the screen monocularly, through the aperture of an electronic shutter. The shutter was closed when the stimulus was presented and opened when the blank field was presented. In this way, if visible phosphor persistence existed, the subjects would be able to distinguish trials in which a picture preceded the blank field from catch trials in which it did not. Rapid alternation of the stimulus and the field in each sequence was used to enhance the likelihood of detecting faint persistence of the stimulus, which might not be obvious if a single brief presentation of stimulus and blank were shown. A third condition, presented after the primary conditions were completed, alternated the stimulus with another picture under the same shutter conditions described above, in order to determine whether picture composites could be detected due to visible phosphor persistence.

To address the second question, the same procedure was followed except that the last video frame of the stimulus presentation was replaced by a bright white field (a "bleaching" field). It was hoped that this would eliminate visible persistence of the stimulus, by charging the phosphor across the entire display.

\section{Method}

Subjects. The subjects were 6 student volunteers (undergraduate and graduate students) from the University of Delaware. Each was paid $\$ 4.50$ for participating. Several worked in our lab and were especially motivated to scrutinize the visual display and report everything they could see.

Stimuli. The stimuli were 12 colored magazine photographs of objects that were cut out and photographed in the center of a gray background to create a set of 35 -mm slides (described in Intraub, 1985). The slides were projected using a Kodak Carousel projector, and the images were captured with a Japan Victor Corporation (JVC) CCD color video camera attached to the graphics system (described in the next section). Image resolution was $378 \times 243$ pixels $\times 16$ bits of color. This allows 65,536 different colors, making up for the relatively low spatial resolution and producing a highquality image. Subjectively, the images appeared to be a televised version of the 16-mm filmed sequences used by Intraub $(1985,1989)$.

The centrally positioned pictures ranged in size, with the smallest covering about $14 \%$ of the gray background and the largest covering $49 \%$. The actual pictures were an organ, a suitcase, a chair, a car, a stove, a pair of eyes, a glass, a tractor, a flag, a hot air balloon, a projector, and a truck. Luminance of the pictures was estimated by using a photometer at six different locations on each object (in the case of a particularly small object and a particularly large object, three and nine locations were measured, respectively). For field luminance, eight measurements were taken, distributed around the field. The mean luminance for the color objects in the computer display was $17 \mathrm{fL}$. Luminance of the gray field was $17 \mathrm{fL}$. Luminance of the white "bleaching" field was $24 \mathrm{fL}$. A small lamp dimly illuminated the experimenter's keyboard; it had no measurable effect on display luminance.

Apparatus. Displays were presented on a Mitsubishi (Model FA3415ATK) color monitor, capable of displaying noninterlaced images. These were presented using an IBM-compatible $(386 / 25-\mathrm{MHz})$ computer, equipped with a Truevision ATVista 4-Mb graphics board. The visual angle subtended by the computer display was approximately $6.4^{\circ} \times 9.0^{\circ}$. 
C programming was used in conjunction with Truevision Stage 2.0 (a programming tool comprising a library of $\mathrm{C}$ commands that drive the various features of the ATVista board). The computer was programmed to drive a Uniblitz electronic shutter (Model 225L) with a Uniblitz (Model SD-122B) shutter drive. The shutter's rise and decay times were reported as 5.5 and $4 \mathrm{msec}$, respectively. Subjects viewed the screen with one eye, through the shutter. To ensure that the subjects were viewing the stimuli monocularly, an opaque shield was attached to the shutter so that if the other eye opened, no view of the display was possible. The shield was constructed out of dark cardboard that was cut to fit around the shutter and cover the nonviewing eye.

Measuring timing. To test if actual display duration corresponded to the duration we specified in our software, we used a photocell connected to a Tektronix storage oscilloscope. Black and white fields were alternated continuously on the monitor with durations of 1 video frame each $(60 \mathrm{~Hz}), 5$ video frames each, or 7 video frames each. The oscilloscope was triggered manually while the series of alternating fields continued to be presented. To confirm that the shutter was opening and closing at the appropriate times, the photocell was placed at the aperture of the shutter (where the subject's eye would normally be positioned), and the same procedure was followed. These tests did indeed reveal a timing problem that was corrected prior to running the experiment. ${ }^{1}$

Design. There were four presentation conditions with $12 \mathrm{se}-$ quences in each. These were mixed randomly in a repeated measures design. Each stimulus picture was presented in all four conditions. In the two "standard" conditions, stimulus pictures were alternated with blank fields (either a gray field that was similar to the gray background of the pictures, or a black field). In the "bleaching" conditions, the last video frame of each picture was replaced with a white "bleaching" field, prior to presentation of the gray or black field.

These 48 sequences were intermixed with another 48 sequences that served as "catch trials." The catch trials were identical to the four conditions just described (i.e., gray field or black field, standard presentation or "bleaching" field), except that stimulus pictures were never actually presented-a blank field corresponding to the interspersing field was presented instead (e.g., a gray field replaced the stimulus when the alternating field was gray). These conditions allowed us to obtain a baseline measure of detection responses when no stimulus was actually presented.

One additional condition was always presented at the end of the experiment proper. Twelve sequences were constructed in which a stimulus picture alternated with another picture. Each pair of pictures was selected to minimize overlap between the two objects (e.g., a horizontal object was alternated with a more vertically oriented object). This was done to maximize the likelihood of detecting any picture composites on the background of gray. Six of these sequences contained a "bleaching" field and six were identical except that they did not contain the "bleaching" field. Thus, each subject was presented with 108 sequences in the course of the experiment.

Procedure. The subjects were individually tested. To familiarize them with the stimuli, they viewed each picture for $5 \mathrm{sec}$ and were provided with its name. They then took part in three naming trials in which they named each picture as quickly as possible. Because they also had participated in pilot experiments, the subjects in this experiment were very familiar with the stimuli and procedures.

The subjects viewed the screen through the shutter. They were told that the experimenter would say "Ready, center" at the start of each sequence. This was their cue to focus attention on the fixation point. Two rows of Xs would then flash on the screen, followed by a 500-msec delay and the $1,400-\mathrm{msec}$ sequence. The sequence was accompanied by the clicking of the shutter, which opened and closed six times in rapid succession. The experimental task required the subjects to report anything they saw on the screen, from a flicker of light to a full picture. Five practice trials were followed by the 108 experimental sequences.
The six rapid alternations of the stimulus and the blank field were used to provide a sensitive test of visible phosphor persistence. If there was any visible persistence, it was more likely to be reported under conditions of multiple repetition rather than a single presentation. Each stimulus was presented for $117 \mathrm{msec}$ ( 7 noninterlaced video frames). The blank field was presented for the same duration. The shutter was always closed when the stimulus picture was presented. The shutter opened when the raster began to paint the blank field (or, in the case of the last 12 conditions, when it began to paint the other picture). It then remained open for $83 \mathrm{msec}$ (five frames), closing for the final two frames of the blank field and for the full time that the stimulus appeared. By closing the shutter for the final two frames, we were able to eliminate the possibility that the shutter's decay time would allow stimuli to be partially glimpsed. In this way, the shutter was already fully closed when the stimulus was presented. Therefore, if the subject could see the stimulus, visible phosphor persistence would be the only cause.

\section{Results and Discussion}

Standard conditions. When the pictures alternated with a black field, persistence was strong enough for every subject to be able to identify each stimulus by name, $100 \%$ of the time. When the pictures alternated with a gray field, visible phosphor persistence was reduced such that none of the subjects $(0 \%)$ reported seeing anything but a blank field on those trials.

"Bleaching" conditions. Inserting the "bleaching" field prior to the black field eliminated reports of visible phosphor persistence for all but 1 subject. This subject reported seeing a "faint object" on $50 \%$ of these trials (although he could not name the objects). All other subjects reported seeing nothing in that condition. In the grayfield condition, when the "bleaching" field was added, as before, the subjects never reported anything other than a blank field on the screen.

Catch trials and picture conditions. The subjects never reported seeing a stimulus or any trace of one on the 48 catch trials. Finally, all subjects searched for, but could not detect, composites of two pictures when the picture pairs were used. This was true both with and without the "bleaching" field.

The results of the shutter test showed that when a picture was followed by another picture or by a gray field, no visible persistence was reported. However, when a picture was followed by a black field, visible persistence was strong enough to allow the subjects to correctly identify each of the pictures. In this extreme case, however, interspersing a "bleaching" field minimized, and in most cases eliminated, visible persistence. For this reason, in the following experiment, although target pictures were always followed by other pictures on gray fields, we explored the use of the white "bleaching" field in view of its possible use as an additional safeguard against phosphor persistence.

\section{EXPERIMENT 2}

The results of Experiment 1 indicated that visible phosphor persistence was evident for our stimuli only when each was followed by a black field. The implication of this finding was that visible phosphor persistence would there- 
fore not pose problems for studying temporal migration if we used the stimuli and gray fields that did not yield visible traces in the shutter test. Experiment 2 directly tested this assumption using behavioral measures rather than a subjective report procedure. We directly compared performance on Intraub's (1985) frame-migration task when the sequences were presented using a $16-\mathrm{mm}$ projector and when they were presented using two types of computer displays (standard presentation and presentation with the interspersed "bleaching" field used in Experiment 1).

Experiments involving alphanumeric stimuli have often been conducted using computer displays, both in visual dissociation experiments, such as those described previously, and in related multiple-task RSVP experiments (Broadbent \& Broadbent, 1987; Kanwisher, 1991; Kanwisher \& Potter, 1990; Raymond, Shapiro, \& Arnell, 1992; Reeves \& Sperling, 1986; Sperling \& Reeves, 1980; Weichselgartner \& Sperling, 1987). In contrast, research involving color photographs has relied on single-frame cinematography and mechanical projection (e.g., Intraub, 1985, 1989; Potter, 1976). Photographs of objects and scenes can vary greatly from one stimulus to the next, so that objects don't always overlap the same spatial area - that is, a part of an object may be followed sometimes by a blank area in the next picture and sometimes by a filled area. With alphanumeric stimuli, one has the option of presenting black stimuli on white backgrounds, thus minimizing persistence of a feature of the letter. Effects of visible phosphor persistence would be expected to be more pronounced for color photographs than for alphanumeric stimuli, and might alter performance on a visual dissociation task.

The rationale for Experiment 2 was as follows. If there is visible phosphor persistence, then we should observe a greater frequency of migration errors with the computer displays than with the cinematic display, because, in addition to mental integration errors, there would be actual stimulus composites appearing briefly on the screen. Furthermore, in addition to the frequency of errors, if the two types of presentation techniques are equivalent, the pattern of errors should also be the same.

Intraub (1985) observed a striking bias for reporting the large frame around the immediately preceding picture in the sequence rather than the immediately following picture. This was in contrast to the pattern typically obtained with alphanumeric stimuli, in which migration occurs more frequently to the following pictures in the sequence (e.g., Gathercole \& Broadbent, 1984; Intraub, 1985, Experiment 4; Lawrence, 1971; McLean et al., 1983; Weichselgartner \& Sperling, 1987). There are several theoretical interpretations of why different migration patterns might occur. These raise interesting questions for future experiments on visual dissociation that are beyond the scope of this paper. The important issue for the present research is whether or not the unusual pattern of migration for large-frame detection would replicate. We expected this pattern to replicate when mechanical projection was used; the question was whether it would also replicate in the computer conditions.
Both computer conditions were tested because we were concerned that the repetitive flashing of the "bleaching" field, while providing an additional safeguard against phosphor persistence, might interfere with task performance. The flashing could induce visual masking or provide a distraction, thus breaking the observer's concentration.

\section{Method}

Subjects. The subjects were 60 male and female undergraduates who had elected to participate in the departmental subject pool.

Apparatus. The graphics system used for the two computer conditions was the same as that used in Experiment 1. A 16-mm Visual Instrumentation Corporation data-analyzer projector was used to present the cinematographic displays. ${ }^{2}$ The projector was located in a projection room, and images were rear-projected on a screen in the room with the computer. All subjects were therefore tested in the same room. They faced either the rear-projection screen or the color monitor. The visual angle subtended by both types of displays was approximately $6.4^{\circ} \times 9.0^{\circ}$.

Stimuli. The stimuli were the same 12 color magazine photographs of objects used in Experiment 1. (A different set of $12 \mathrm{ob}-$ jects was used in the practice sequences that preceded the actual experiment). The stimuli and the practice set contained the same pictures as those used by Intraub (1985), with the exception of one picture in the practice set.

As described in Experiment 1, the computer images appeared, subjectively, to be of the same quality as projected images. Luminance of the computer and film displays differed, however, in that the computer yielded a brighter image. Luminance for the computer sequences was as described in Experiment 1. For film presentation, mean luminance for the same pictures was $3 \mathrm{fL}$.

Design. All 12 pictures served as the target picture (the one with the black frame) twice, except for the suitcase and the glass. The suitcase was the target picture only once and the glass was the target three times. The reason for this discrepancy was a filming error carried over from Intraub (1985). It was carried over to the present research to allow for an accurate replication. The positions in the sequence for the target picture were equally distributed in Positions 2 , 5,8 , and 11 . The pictures that flanked a given target picture were reversed the second time that target picture was presented.

The same 24 sequences were presented using the computer display without the "bleaching" field, the computer display with the "bleaching" field, and the projector. Twenty subjects participated in each condition. Presentation rate in the projector condition was $114 \mathrm{msec} /$ picture. Due to the fixed refresh time of video displays, the presentation rate in the two computer conditions was $117 \mathrm{msec} /$ picture (seven video frames). In the "bleaching" field condition, the last video frame associated with the stimulus was replaced by a bright white field (i.e., six video frames of the stimulus and one of the "bleaching" field). Stimulus onset asynchrony was therefore the same in both computer conditions.

Procedure. The subjects were tested individually. They were familiarized with the pictures and their names following the same procedure as in Experiment 1. As in Intraub (1985), to familiarize them with the black frame, the pictures were presented again, this time with the frame around each one. Again, the subjects were told that the experimenter would say "Ready, center" at the start of each sequence to cue them to focus attention on the fixation point in the center of a gray field. Two rows of Xs would then flash on the screen, followed by a $500-\mathrm{msec}$ delay and the sequence. The experimental task required the subjects to respond with the name of the picture enclosed by the black frame as soon as they saw the frame. The subjects were instructed to provide confidence ratings of their responses (sure, pretty sure, not sure, or guess). This procedure was carried out for the practice picture set and the practice sequences, and was then carried out for the stimulus picture set and the 24 experimental sequences. 


\section{Results and Discussion}

The subjects' confidence ratings in each condition were similar to those obtained in Intraub's (1985) experiments and are presented in Table 1 . The subjects failed to respond on $1.3 \%$ or fewer of the trials in each condition ("misses"). As in the previous research, all analyses were conducted on high-confidence trials (those with ratings of sure or pretty sure). The percentage of high-confidence responses that were correct (hits) and those that were incorrect at each serial position are shown in Table 2.

All three conditions replicated the pattern of results reported by Intraub (1985). The subjects made numerous high-confidence migration errors, usually reporting the immediately preceding or immediately following picture as the one with the frame $(-1$ and +1 pictures, respectively). In all conditions, fewer than $8 \%$ of all highconfidence error responses fell outside this window.

The important comparisons were between the standard computer condition (no "bleaching" field) and the projector condition, and the two computer conditions. Nonorthogonal planned comparisons conducted on the proportion of correct target detections (hit rate) revealed no significant difference between the standard computer condition and the projector condition $[F(1,57)=1.19]$. Although there appeared to be an increase in accuracy when the "bleaching" field was added to the computer display, this difference between the two computer conditions did not reach significance $\left[F(1,57)=2.43, M S_{\mathrm{e}}=\right.$ $550.20, p<.13]$.

Similar to Intraub's $(1985,1989)$ previous research, there was a bias for reporting the immediately preceding picture in the sequence. Wilcoxon tests showed this bias to be significant (at $p<.01)$ in each condition $(T=-6$, $n=19 ; T=0, n=17 ; T=9, n=16$; in the projector, computer, and computer with "bleaching" field conditions, respectively). To consider the degree of bias, difference scores were obtained for the three conditions by subtracting the proportion of errors to later pictures in the sequence from the proportion of errors to the preceding pictures. The mean difference scores are shown in Table 3. Nonorthogonal planned comparisons showed that the size of the difference score (i.e., the size of the bias to report earlier pictures) did not change when the standard computer condition was compared with the projector condition $(F<1)$ or when it was compared with the computer condition in which the "bleaching" field was presented $[F(1,57)=1.88]$.

The pattern of temporal migration was unaffected by presentation mode. Subjects did not make more errors

Table 1

Percentage of Responses at Each Confidence Level as a Function of Presentation Condition

\section{Confidence}

\begin{tabular}{lcccc}
\cline { 2 - 5 } \multicolumn{1}{c}{ Condition } & Sure & Pretty Sure & Not Sure & Guess \\
\hline Projector & 9 & 40 & 44 & 7 \\
Computer & 9 & 49 & 35 & 7 \\
Computer (IBF) & 7 & 44 & 43 & 4 \\
\hline
\end{tabular}

Note-IBF, interspersed "bleaching" fields.
Table 2

Mean Percentage of High Confidence (Sure and Pretty Sure)

Responses Reporting the Frame on the Correct Picture

(Hit), Preceding Pictures (-), and the Following

Pictures (+), for Each Presentation Condition (Rounded to the Nearest Whole Number)

\begin{tabular}{lrrrrrrr}
\hline & \multicolumn{6}{c}{ Position of Reported Picture in Sequence } \\
\cline { 2 - 8 } \multicolumn{1}{c}{ Condition } & -3 & -2 & -1 & Hit & +1 & +2 & +3 \\
\hline Projector & 1 & 0 & 41 & 51 & 5 & 0 & 0 \\
Computer & 1 & 1 & 35 & 59 & 3 & 0 & 1 \\
Computer (IBF) & 5 & 0 & 24 & 71 & 3 & 0 & 1 \\
\hline
\end{tabular}

Note-IBF, interspersed "bleaching" fields.

when viewing the display on the monitor than they did with the projection screen, and the pattern of results (i.e., a bias toward reporting preceding pictures) was upheld. The addition of a briefly flashed white "bleaching" field clearly did not result in a performance decrement; if anything, subjects in this condition tended to be slightly more accurate in target detection. If future research shows this difference to be reliable, possible reasons might be that (1) the interspersed flashes may heighten awareness, or (2) the white field may be interpreted by the visual system as a new event (or may act as a mask) so that it reduces integration errors between temporally adjacent pictures in the visual/conceptual short-term store. What can be determined from the current experiment, however, is that the addition of the "bleaching" field does not hurt performance or change the general pattern of errors in a visual dissociation task.

\section{EXPERIMENT 3}

Experiment 3 followed up the findings of Experiment 2, using three different rates of presentation $(100,117$, and $133 \mathrm{msec}$ per picture). Film presentation does not allow for very fine-grained differences in presentation rate. Computer presentation, however, allowed us to readily compare performance at presentation rates in which the stimulus onset asynchrony could be changed in 17-msec increments. According to the visual integration model described earlier, frame migration is influenced by the processing time needed to integrate the components of a display and the speed at which the pictures are presented. Therefore, according to this model and, in fact, to most models that would attribute the effect to on-line processes (rather than to a response error occurring in memory following presentation), these subjectively similar-looking presentation rates should yield a greater frequency of high-confidence migration errors as presentation rate is increased.

\section{Method}

Subjects. The subjects were 32 undergraduate volunteers from the same population as in Experiment 2.

Stimuli and Apparatus. The same apparatus and the same sequences of digitized color photographs were used as in Experiment 2.

Design and Procedure. The subjects were randomly assigned to one of three conditions: slow (133 msec/picture), medium (117 msec/ 
Table 3

Mean Difference Score as a Function of Presentation Condition

\begin{tabular}{lll}
\hline \multicolumn{1}{c}{ Condition } & $M$ & $S D$ \\
\hline Projector & 37 & 32 \\
Computer & 32 & 22 \\
Computer (IBF) & 21 & 24 \\
\hline
\end{tabular}

Note-IBF, interspersed "bleaching" field.

picture), or fast ( $100 \mathrm{msec} /$ picture). There were 10 subjects in the slow condition and 11 each in the medium and fast conditions. The instructions and procedure were the same as in Experiment 2. The "bleaching" field was not presented. Note that the medium condition was a replication of the standard computer condition in Experiment 2.

\section{Results and Discussion}

The same visual dissociation pattern reported earlier for the large-frame condition was again replicated in this experiment; the subjects frequently made high-confidence errors involving the immediately preceding and following pictures in the sequence. As with previous large-frame conditions, when high-confidence errors were made, they were more frequently made to previous pictures in the sequence (negative migration). Table 4 shows the percentage of responses associated with each confidence rating for each condition. Table 5 shows the percentage of highconfidence responses as a function of the position of the reported picture in the sequence. A one-way analysis of variance showed that the number of high-confidence (sure and pretty sure) responses did not differ significantly across conditions $\left[F(2,29)=1.59, M S_{\mathrm{e}}=19.70, \mathrm{n} . \mathrm{s}\right]$. Although subjects did not become less confident as rate increased, the proportion of their high-confidence responses that were correct (hits) decreased as presentation rate increased $\left[F(2,29)=6.18, M S_{\mathrm{e}}=2,355.16, p<.006\right]$. The subjects were not simply unsure at the faster rates; they were just as confident, but made a greater number of perceptual integration errors.

\section{GENERAL DISCUSSION}

When the field illumination is adjusted appropriately, computer video displays may be used to conduct visual perception experiments requiring brief successive presentation of digitized color photographs. Using a multiplepresentation shutter test, Experiment 1 showed that whether or not there was visible phosphor persistence reported by the subjects depended on the luminance of the display that followed the stimulus. When the pictures were followed by a gray field, or by another color picture centered on the gray field, no indication of visible phosphor persistence was obtained. However, when a black field followed it, persistence was strong enough to allow subjects to identify which digitized photograph had left the trace. For this reason, care must be taken when using pictures that contain dark, relatively homogeneous areas that might allow visible phosphor persistence to contaminate results. It would be advisable to conduct a shutter test for each new stimulus set to be used in experiments such as these.
Although the results of the shutter test were encouraging, subjective report alone would not be sufficient to allay concerns about this aspect of computer presentation. Experiments 2 and 3 were performed to determine whether subjects' behavioral data would converge on the same conclusion. In Experiment 2, a direct comparison was made between subjects' performance on a visual dissociation task with photographs using mechanical projection (cinematography, as in Intraub, 1985) and computer presentation. Intraub's research was replicated, and the same pattern of errors and similar levels of accuracy and confidence were obtained in the projector and computer conditions. In no instance did the computer presentations yield lower accuracy, as would be expected if visible phosphor persistence were causing fleeting stimulus composites on the screen.

Experiments 1 and 2 also showed that once the stimulus set had been screened using the shutter test, the addition of a white "bleaching" field following each picture did not alter the general pattern of results or cause poor performance. If anything, subjects were more accurate with the interspersed field. This tendency toward less migration might indicate that the visual system interprets the flash as a new visual event, thus limiting integration errors between pictures. This issue in itself raises interesting questions for future research, but unless one is directly testing these possibilities, care should be taken in introducing the "bleaching" field when conducting visual dissociation experiments. However, in experiments requiring single tachistoscopic exposures of pictures, the interspersed field would be useful as an additional safeguard against phosphor persistence.

Finally, unlike cinematographic presentation, computer presentation allowed us to alter presentation rate in terms of very small increments (one video frame; $16.667 \mathrm{msec}$ ), and to study the effect of these small changes on temporal migration - changes that might not be subjectively noticeable but that would be expected to affect visual integration. Experiment 3 showed that, relative to the baseline rate of $117 \mathrm{msec}$ per picture, an increase or decrease of one video frame did not affect subjects' confidence ratings but did significantly affect the frequency of temporal migration errors. Whereas the baseline rate was associated with $60 \%$ correct detections, a decrease of $17 \mathrm{msec}$ yielded only $42 \%$ correct and an increase of $17 \mathrm{msec}$ yielded $72 \%$ correct.

In conclusion, the results indicate that computer technology may be used to conduct research on visual perception that requires rapid, successive presentation of digi-

Table 4

Percentage of Responses as a Function of Confidence for Each Presentation Rate

\begin{tabular}{lcccccc}
\hline \multicolumn{2}{c}{ Rate } & & \multicolumn{4}{c}{ Responses } \\
\cline { 1 - 4 } \cline { 5 - 7 } Condition & $\begin{array}{c}\text { Milliseconds } \\
\text { per Item }\end{array}$ & & Sure & $\begin{array}{c}\text { Pretty } \\
\text { Sure }\end{array}$ & Not Sure & Guess \\
\hline Fast & 100 & & 14 & 44 & 28 & 10 \\
Medium & 117 & & 8 & 37 & 42 & 10 \\
Slow & 135 & & 8 & 45 & 35 & 10 \\
\hline
\end{tabular}


Table 5

Mean Percentage of High-Confidence (Sure and Pretty Sure)

Responses Reporting the Frame on the Correct Picture

(Hit), Preceding Pictures (-), and Following

Pictures $(+)$ at Each Presentation Rate

\begin{tabular}{|c|c|c|c|c|c|c|c|c|}
\hline \multicolumn{2}{|c|}{ Rate } & \multirow{2}{*}{\multicolumn{7}{|c|}{ Position of Reported Picture in Sequence }} \\
\hline \multirow[b]{2}{*}{ Condition } & \multirow{2}{*}{$\begin{array}{l}\text { Milliseconds } \\
\text { per Item }\end{array}$} & & & & & & & \\
\hline & & -3 & -2 & -1 & Hit & +1 & +2 & +3 \\
\hline Fast & 100 & 0 & 1 & 54 & 42 & 2 & 0 & \\
\hline Medium & 117 & 1 & 0 & 35 & 60 & 3 & 2 & \\
\hline Slow & 133 & 0 & 0 & 28 & 72 & 0 & 0 & \\
\hline
\end{tabular}

Note-Rates are rounded to the nearest whole number.

tized color photographs. Specifically, this seems to be the case for visual dissociation research, which would be contaminated by displays with visible phosphor persistence. The application of computer graphics technology to research on picture perception will allow for much more refined tests of visual integration, including the ability to alter photographs of scenes to study the possible effect on scene structure on temporal migration (see Intraub, 1989). Hopefully, it will foster more research on complex pictorial stimuli.

\section{REFERENCES}

Avons, S. E., \& PhILlips, W. A. (1980). Visualization and memorization as a function of display time and poststimulus processing time. Journal of Experimental Psychology: Human Learning \& Memory, $6,407-420$.

Broadbent, D. E., \& Broadbent, M. H. P. (1987). From detection to identification: Response to multiple targets in rapid serial visual presentation. Perception \& Psychophysics, 42, 105-113.

BURR, D. A. (1980). Motion smear. Nature, 284, 164-165.

Coltheart, M. (1980). Iconic memory and visible persistence. Perception \& Psychophysics, 27, 183-228.

DiLollo, V., \& Dixon, P. (1988). Two forms of persistence in visual information processing. Journal of Experimental Psychology: Human Perception \& Performance, 14, 671-681.

Dixon, P. (1991). Tachistoscopic display using color look-up table display devices. Behavior Research Methods, Instruments, \& Computers, 23, 380-386.

FARRELL, J. E. (1984). Visible persistence of moving objects. Journal of Experimental Psychology: Human Perception \& Performance, 10, 502-511.

Farrell, J. E., Pavel, M., \& Sperling, G. (1990). The visible persistence of stimuli in stroboscopic motion. Vision Research, 30, 921-936.

Gathercole, S. E., \& Broadbent, D. E. (1984). Combining attributes in specified and categorized target search: Further evidence for strategic differences. Memory \& Cognition, 12, 329-337.

INTRAUB, H. (1984). Conceptual masking: The effects of subsequent visual events on memory for pictures. Journal of Experimental Psychology: Learning, Memory, \& Cognition, 10, 115-125.

INTRAUB, H. (1985). Visual dissociation: An illusory conjunction of pictures and forms. Journal of Experimental Psychology: Human Perception \& Performance, 11, 431-442.

INTRAUB, H. (1989). Illusory conjunctions of forms, objects, and scenes during RSVP search. Journal of Experimental Psychology: Learning, Memory, \& Cognition, 15, 98-109.

IRWIN, D. E., YANTIS, S., \& JoNIDES, J. (1983). Evidence against visual integration across saccadic eye movements. Perception \& Psychophysics, 34, 49-57.

JoNiDES, J., IRWIN, D. E., \& YANTIS, S. (1983). Failure to integrate information from successive fixations. Science, 222, 188.
KANWISHER, N. (1991). Repetition blindness and illusory conjunctions: Errors in binding visual types with visual tokens. Journal of Experimental Psychology: Human Perception \& Performance, 17, 404-421.

Kanwisher, N., \& PotTer, M. (1990). Repetition blindness: Levels of processing. Journal of Experimental Psychology: Human Perception \& Performance, 16, 30-47.

LAWRENCE, D. H. (1971). Two studies of visual search for word targets with controlled rates of presentation. Perception \& Psychophysics, 10, 85-89.

LofTus, G. R., Duncan, J., \& Gehrig, P. (1992). On the time course of perceptual information that results from a brief visual presentation. Journal of Experimental Psychology: Human Perception \& Performance, 18, 530-549.

Mclean, J. P., Broadbent, D. E., \& Broadbent, M. H. P. (1983). Combining attributes in rapid serial visual presentation tasks. Quarterly Journal of Experimental Psychology, 35A, 171-186.

NeISSER, U. (1967). Cognitive psychology. New York: Appleton.

Paredes, D. R., Miller, K. F., \& Creeger, C. (1990). Graphic precision: Controlling stimulus displays on IBM PC-compatible computers. Behavior Research Methods, Instruments, \& Computers, 22, 319-322.

PotTer, M. C. (1976). Short-term conceptual memory for pictures. Journal of Experimental Psychology: Human Learning \& Memory, 2, 509-522.

Raymond, J. E., Shapiro, K. L., \& Arnell, K. M. (1992). Temporary suppression of visual processing in an RSVP task: An attentional blink? Journal of Experimental Psychology: Human Perception \& Performance, 18, 849-860.

RaYNER, K., \& PollatseK, A. (1983). Is visual information integrated across saccades? Perception \& Psychophysics, 34, 39-48.

REEVES, A., \& SPERLING G. (1986). Attention gating in short-term visual memory. Psychological Review, 93, 180-206.

SPERLiNG, G., \& REEVES, A. (1980). Measuring the reaction time of a shift of visual attention. In R. Nickerson (Ed.), Attention and performance VIII (pp. 347-360). Hillsdale, NJ: Erlbaum.

WEICHSELGaRTNER, E., \& SPERLING, G. (1987). Dynamics of automatic and controlled visual attention. Science, 238, 778-780.

\section{NOTES}

1. Initial testing revealed a timing problem that involved two different sources. One concerned an undocumented characteristic of the SetPanPos command in the Truevision Stage 2.0 programming library. Its purpose is to reposition the viewing port of the screen buffer. This command includes, within its program, a vertical blank wait command (VBLWait), which was not documented. Our programmer therefore had included a separate VBLWait command. Thus, two successive commands of this type resulted in the addition of an unexpected, but systematic, increase in presentation time. Technical support at Truevision, the developers of both the ATVista card and the Stage 2.0 software, supported our programmer's conclusion regarding SetPanPos.

The second problem involved an asynchronous relationship between the computer's Intel 386 processor and the AT-Vista graphics card, which has its own Texas Instruments processor. Although the order of commands in a DOS C program dictates the execution order (discounting interrupts), the presence of the two processors resulted in a reversal of execution order of two commands, causing the shutter to open and close one frame too soon. This consistent error was compensated for by reordering the code.

2. This type of projector has the same chassis as a typical $16-\mathrm{mm}$ projector but is altered to allow for carefully controlled film speed, as well as a wider range of speeds, without compromising luminance. This type of projector was typically used in rapid visual presentation research and research requiring animation before computer technology was available.

(Manuscript received December 29, 1994; revision accepted for publication June 1, 1995.) 